\title{
3D Modelling of Temperature Dependent Power Distribution During Microwave Annealing of Doped c-Si
}

\author{
S D Das \\ Center of Excellence for Green Energy and Sensor Systems, BESU, India
}

\begin{abstract}
Recrystallized silicon solar cells using microwave annealing will require adequate modelling of microwave power distribution to predict the uniformity of re-crystallization of silicon, specially at high temperatures. Since, recrystallization will have to be carried out of doped silicon, doping dependence of such processes at high temperatures is also very important. This work reports modelling results for temperature and doping dependence of microwave spatial power distribution in $c$-Si wafer during microwave annealing. Temperature dependence was introduced through bandgap, effective mass and lattice scattering terms in Drude model of free-carrier concentration. Dependence of doping was introduced through free-carrier dependent complex dielectric function. Absorption of power was then calculated from the complex part of the dielectric constant. The 3D Modelling was carried out using Exact Finite Difference Method. All results presented in this paper are for Industrial, Scientific and Medical (ISM) frequency of $2.45 \mathrm{GHz}$.
\end{abstract}

Keywords: solar cells, 3D, microwave, annealing, doping

\section{INTRODUCTION}

Sustainable technology is key to long term advancement of renewable energy sector. Microwave heating is one such sustainable technology which could be adopted for manufacturing of solar cells. Many such schemes have been identified in the recent past and recrystallized silicon solar cell using microwave annealing is one such technique [1]. The concept calls for ion implantation of doped c-Si wafer to form p-n junction. Microwave annealing should then be carried out for recrystallization and implantation damage removal [2-3]. Such high temperature annealing process may also activate the dopants depending upon temperature achieved inside the sample and doping species. To predict the spatial uniformity of recrystallization process using microwave annealing, spatial distribution of absorbed microwave power by silicon needs to be modelled accurately. To model recrystallized silicon solar cells, understanding of the processes in implanted region and doped c-Si substrate region separately is necessary; since based on spatial distribution of material property such as dielectric function selective heating due to microwave absorption will take place. Full system 3D modelling technique using Exact Finite Difference Technique (eFDM) of doped c-Si at 300K has been reported elsewhere [4], which describes the importance of cavity dimensions on such procedure.

In this work, first temperature and doping dependence of material dielectric function will be introduced through Drude's model; followed by reporting of results from full system 3D modelling of spatial distribution of absorbed microwave power in doped c-Si substrate using eFDM technique. Dependence of such process on temperature between $300 \mathrm{~K}-800 \mathrm{~K}$ at different doping levels of c-Si substrate will be presented.

II. Modelling Temperature DePendence OF DiELECTRIC FunCtion In DOPED SiLICON In non-polar semiconductors such as c-Si the mechanism of absorption at ISM frequency is primarily free-carrier absorption which depends upon doping levels and temperature of the material. Microwave power absorbed by non-magnetic materials is directly proportional to relative imaginary part of complex dielectric function $\varepsilon_{c}^{r}$ [5]. The complex dielectric function ${ }^{\mathcal{E}}$ for c-Si dependent on free-carrier absorption is given by Drude's model $[6,7]$ :

$$
\varepsilon=\varepsilon_{r}+i \varepsilon_{c} \square \varepsilon_{\text {freccarrier }}=\varepsilon_{\text {core }}+\frac{4 \pi i}{\omega}\left[\frac{n_{e} e^{2} \mathbf{T}_{e}}{m_{e}\left(1-i \omega \mathbf{T}_{e}\right)}+\frac{n_{h} e^{2} \mathbf{T}_{h}}{m_{h}\left(1-i \omega \mathbf{T}_{h}\right)}\right]
$$

Drude model calculates complex dielectric function from complex conductivity of the material and conductivity of a material is related to total free carrier concentration which depends on doping concentration and temperature via Fermi level. Total free carrier concentration is dependent on thermally generated and photogenerated carriers. In this work photo-generation is neglected as at c-Si bandgap this could be neglected for microwave annealing under dark conditions. The Fermi level depends directly on doping concentration and temperature, and to improve accuracy of the modelling temperature dependence of effective masses and 
bandgap have been included as well which affects Fermi level indirectly. Temperature dependant effective masses also directly influence the dielectric function and they have been modelled using Green et. al. model [8]:

$$
\begin{aligned}
& \frac{m_{e}(T)}{m_{0}}=6^{2 / 3}\left[\left(C \frac{E_{g}(0)}{E_{g}(T)}\right)^{2} \frac{m_{l}}{m_{0}}\right]^{1 / 3)} \\
& \frac{m_{h}(T)}{m_{0}}=\left(\frac{a+b T+c T^{2}+d T^{3}+e T^{4}}{1+f T+g T^{2}+h T^{3}+i T^{4}}\right)^{2 / 3}
\end{aligned}
$$

where, $m_{e}$ and $m_{h}$ are effective mass of electron and hole, respectively. For temperature dependence bandgap standard Varshni equation has been used [9].

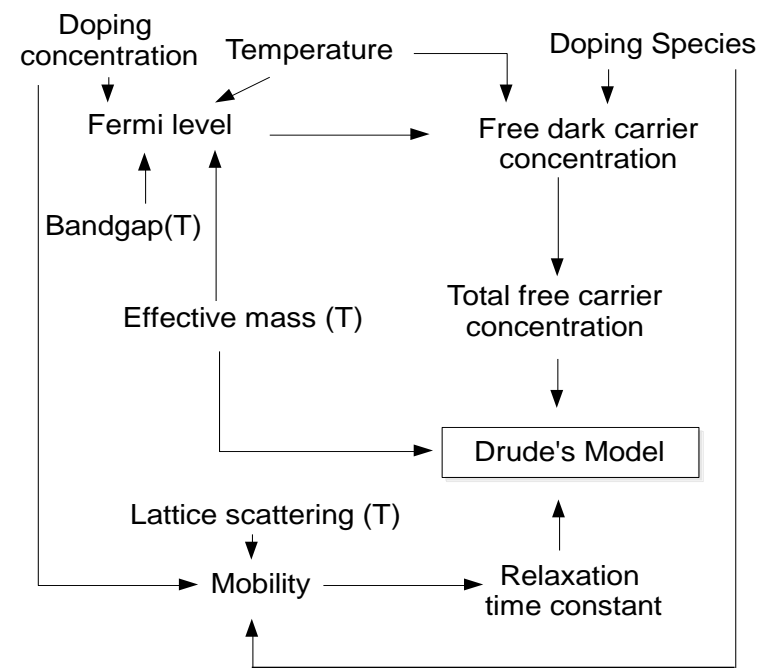

Fig.1. Introduction of doping and temperature dependence to Drude's model of dielectric function.

The temperature and doping dependence of relaxation time constants for minority and majority carrier ( ${ }^{T_{e}}$ and $\mathbf{T}_{h}$ ) are obtained from Reggiani et. al. temperature and doping dependent mobility model for c-Si [10] :

$$
\mu\left(N_{D}, N_{A}, T\right)=\mu_{o}+\frac{\mu_{L}-\mu_{0}}{1+\left(\frac{N_{D}}{C_{r 1}}\right)^{\alpha_{1}}+\left(\frac{N_{A}}{C_{r 2}}\right)^{\alpha_{2}}}-\frac{\mu_{1}}{1+\left(\frac{N_{D}}{C_{s 1}}+\frac{N_{A}}{C_{s 2}}\right)^{-2}}
$$

where, $\mu_{L}$ gives the temperature dependant lattice scattering of free-carrier. Both relaxation time constants and free carrier concentration depends on the doping species like phosphorous (P), arsenic (As) or boron (B). For more accurate results bandgap narrowing due to doping should be introduced. Change in bandgap due to high doping is of the order of few meV and only severely affects ionised impurity concentration at low temperatures and at very high doping levels. As this work focuses on high temperatures, effects of doping dependence on bandgap will be ignored for this publication.

\section{RESULTS AND DISCUSSIONS}

Variation of complex part of relative dielectric function with doping concentration and temperature as described by (1) to (4) is shown in the Fig. 3. The dopant species is phosphorous (P) and important modelling values are tabulated in Table 1. From Fig. 3, it is evident that ${ }^{\varepsilon_{c}^{r}}$ values increases with donor concentration first and then rate of increase slows down. The slowing down sets in at higher temperatures for higher values of doping concentration. This is due to the fact that at a given temperature and at high values of doping concentration, number of ionized dopants tend to saturate according to governing distribution of carrier over thermal energy and with the increase in temperature this saturation effect starts at higher values of doping 
concentrations. This fact is clearly reflected in ${ }^{r}$ values as $\varepsilon_{c}^{r}$ is directly dependant on free carrier concentration in Drude model.

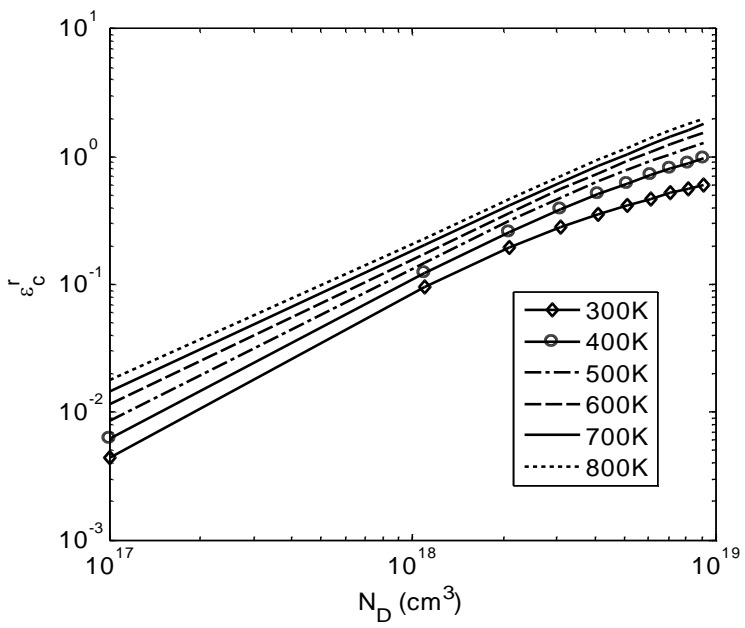

Fig. 2 Imaginary part of relative dielectric function ${ }^{\varepsilon_{c}^{r}}$ as a function of donor concentration $\left(N_{D}\right)$ for temperatures $T=300 \mathrm{~K}$ to $800 \mathrm{~K}$. $(f=2.45 \mathrm{GHz})$.

Table 1. Modelling values for complex dielectric constant

\begin{tabular}{|c|c|}
\hline Parameters & Values \\
\hline$\varepsilon_{\text {core }}$ & 11.9 \\
\hline$\mu_{L}=\mu_{\max }\left(\frac{T}{300}\right)^{\left(-\mathrm{\gamma}+\frac{c * T}{300}\right)}$ & $\mu_{L}=1441\left(\frac{T}{300}\right)^{\left(-2.45+\frac{0.07 * T}{300}\right)}$ \\
\hline $\mathrm{T}_{e}=m_{e} m_{o} \frac{\mu_{e}}{q}$ & $\mathrm{~T}_{e}=9.1 \times 10^{-29} 1.26 \times 10^{-8} \frac{\mu_{e}}{q}, \mu_{e}$ is given by (4) \\
\hline$N_{a}$ & $1 \times 10^{14} \mathrm{~cm}^{-3}$ \\
\hline
\end{tabular}

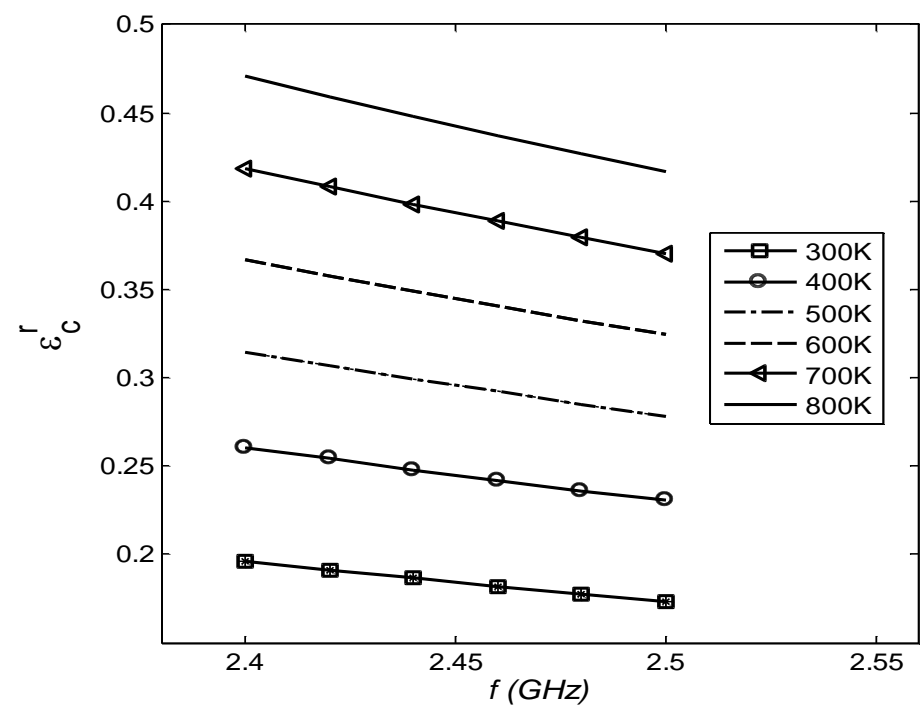

Fig. 3 Imaginary part of relative dielectric function $\varepsilon_{c}^{r}$ as a function of frequency $(f)$ with temperature as parameter from $300 \mathrm{~K}$ to $800 \mathrm{~K}$.

In a household microwave oven, the frequency of operation is not limited to $2.45 \mathrm{GHz}$, but has a range defined by $\pm 50 \mathrm{MHz}$. Fig. 3 shows typical variation of $\varepsilon_{c}^{r}$ values with frequency at various temperature values between 
$300 \mathrm{~K}$ to $800 \mathrm{~K}$ for donor concentration $\left(\mathrm{N}_{\mathrm{D}}\right)$ of $2 \times 10^{18} \mathrm{~cm}^{-3}$. These results indicate $\varepsilon_{c}^{r}$ is more sensitive to $\mathrm{N}_{\mathrm{D}}$ as compared to temperature of c-Si and frequency of operation.

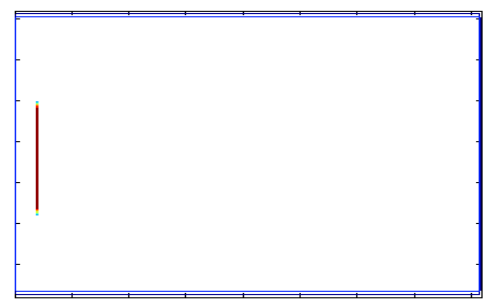

Fig. 4. Full system microwave power distribution (vertical cross section rotated $90^{\circ}$ clockwise) with c-Si wafer placed vertically at the middle left corner. $f=2.45 \mathrm{GHz}$

The full system modelling of absorbed microwave power was carried out by solving the Helmholtz equation using 3D eFDM technique[3]. In this work full system comprised of a c-Si wafer placed inside a metal cavity without any material support. Dimensions of the oven cavity was kept as earlier, while dimensions of c-Si wafer was $10.5 \times 10.5 \times 0.06 \mathrm{~cm}^{3}$. A dense mesh was used in the $\mathrm{z}$-direction to maintain reasonable number of nodes for solving Helmholtz equation inside the wafer, corresponding points per unit wavelength was maintained at $(15,15,1000)$ for $(x, y, z)$ directions. This gave rise to matrix with $1,998,792$ number of elements. Microwave power distribution results for full system are shown in the Fig. 6. From this figure it is evident that most of the energy is concentrated inside the wafer. From relative peak absorption power curves (Fig. 7(a) \& 7(b)) of c-Si, it can be inferred that absorption increases by an order of magnitude if doping density is increased by an order of magnitude, however increase in absorption power is much smaller when doping density is kept constant and temperature of wafer is increased. This is due to little change in $\varepsilon_{c}^{r}$ with temperature, as is evident from Fig. 2. Fig. 5(c) provides relative peak power spectrum of the absorbed power inside the wafer at $800 \mathrm{~K}$ and $\mathrm{N}_{\mathrm{D}}=$ $1 \times 10^{18} \mathrm{~cm}^{-3}$, showing some frequencies are well supported while others are not and peak of absorption is close to $f=2.45 \mathrm{GHz}$.

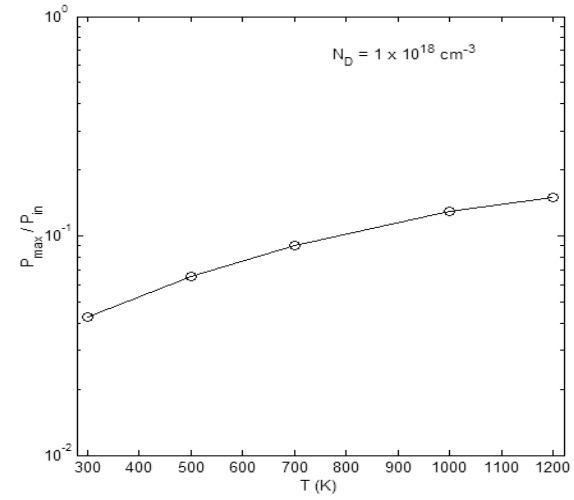

(a)

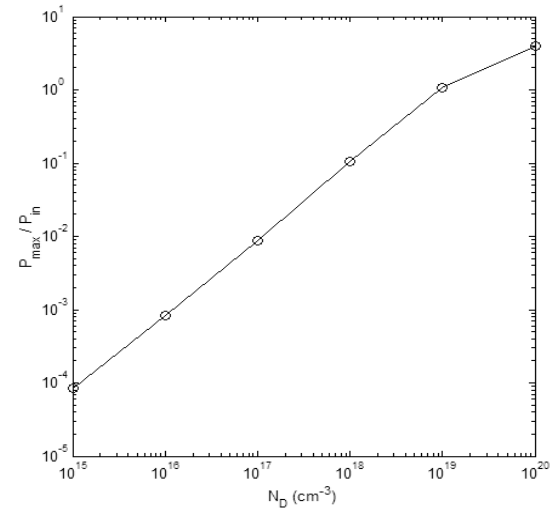

(b)

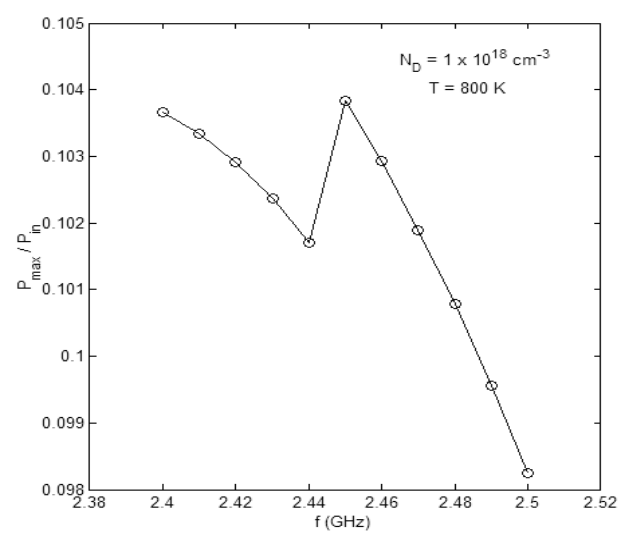


(c)

Fig 5. Relative peak power absorbed with in c-Si wafer (a) as function of temperature ( $f=2.45 \mathrm{GHz})$; $(b)$ as function of doping density at $300 \mathrm{~K}(f=2.45 \mathrm{GHz})$ and (c) as a function of frequency.

The results for full system spatial distribution of microwave power absorption in c-Si wafer is shown in Fig. 6. From these figures it is evident that even at high temperature and high doping density the spatial distribution is not affected by these parameters and dominant parameters are dimensions of sample and microwave cavity. As suggested by Fig 5(c), peak absorption characteristics may be obtained at particular frequency for given dimension of sample and microwave cavity.

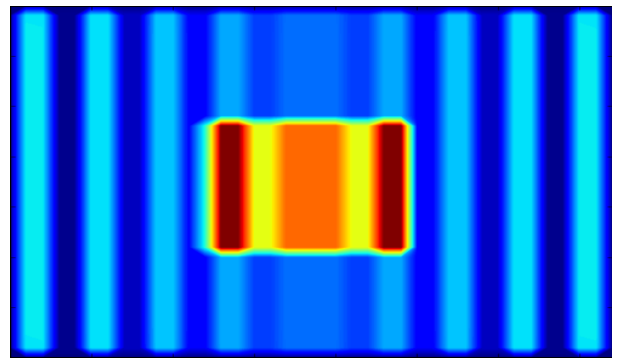

(a)

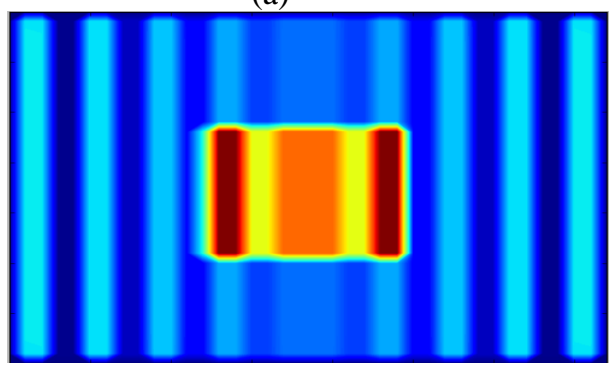

(a)

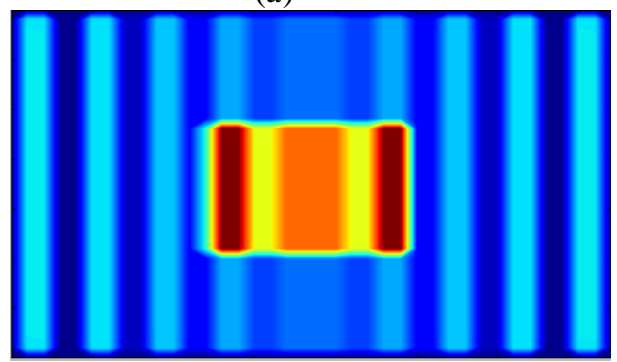

(a)

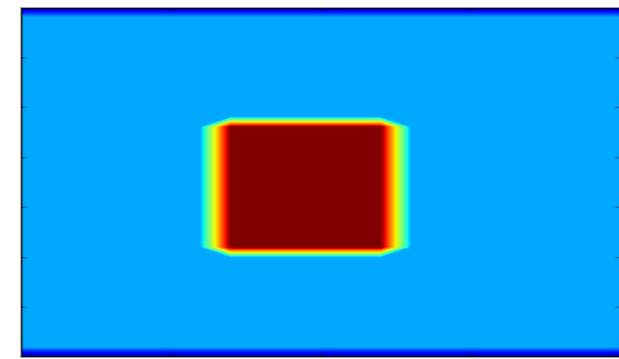

(b)

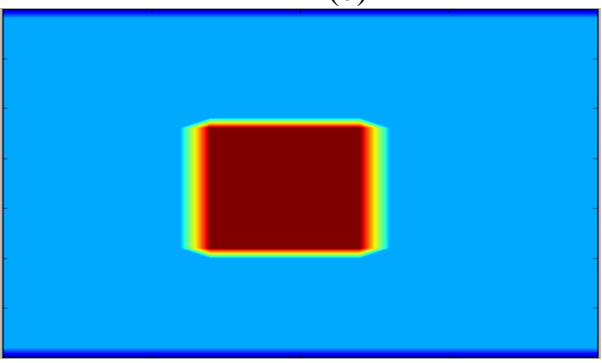

(b)

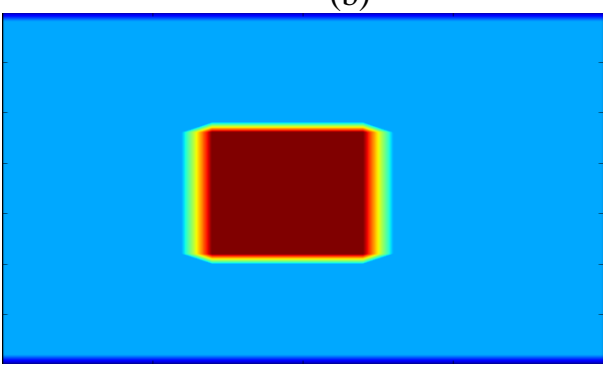

(b)

Fig 6. Microwave power distribution; (a) horizontal cross section and (b) vertical cross section (magnified and rotated $90^{\circ}$ clockwise) for $f=2.45 \mathrm{GHz}, \mathrm{T}=800 \mathrm{~K}$ at (1) $\mathrm{N}_{\mathrm{D}}=1 \times 10^{15} \mathrm{~cm}^{-3}$, (2) $\mathrm{N}_{\mathrm{D}}=1 \times 10^{18}$ $\mathrm{cm}^{-3}$ and $(3) \mathrm{N}_{\mathrm{D}}=1 \times 10^{20} \mathrm{~cm}^{-3}$.

\section{CONCLUSION}

In conclusion, 3D modelling results for spatial distribution of microwave power in phosphorous doped $\mathrm{c}-\mathrm{Si}$ wafer has been presented. Temperature and doping dependence in microwave annealing of c-Si wafer was introduced through Drude's model of complex dielectric constant. Results from modelling show that temperature has weak influence on complex part of dielectric function while doping do have significant influence on dielectric function but spatial distribution is not dominated by the two parameters. Thus uniformity issues of spatial distribution of absorbed microwave power at $f=2.45 \mathrm{GHz}$ is not dominated by doping levels and temperature of c-Si wafer. Further work on uniformity issues will require accurate modelling of ion-implanted region of the recrystallized silicon solar cells.

\section{Acknowledgements}

This work was carried out as Research Associate at CEGESS, BESU under the DST Project entitled 'Solar Photovoltaic Hub at BESU". Author would like to thank institute, university and funding agency for their full support and encouragement. 


\section{REFERENCES}

[1] S. D. Das, Prospects of Microwave Heating in Silicon Solar Cell Fabrication - A Review, IOSR-JEEE, 6(3), 2013, 28-38.

[2] R. N. P. Vemuri, M. J. Garde, N. D. Theodore, and T. L. Alford, Dopant Activation in Arsenic -Implanted Si by SusceptorAssisted Low-Temperture Microwave Anneal, IEEE Electron Device Letters 32, 2011, 1122-1124.

[3] J. N. Lee, Y. W. Choi, B. J. Lee and B. T. Ahn, Microwave-induced low-temperature crystallization of amorphous silicon thin films, Journal of Applied Physics, 82(6), 1997, 2918-2921.

[4] S. D. Das, Full wafer 3D modelling of power distribution during microwave annealing of doped c-Si, IOSR-JEEE, 9 (1-IV), 2014, 68-76

[5] T Basak, Role of lateral and radial irradiations on efficient microwave processing of food cylinders, Chemical Engineering Science, 62, 2007, $3185-3196$.

[6] D. C. Dibben, Numerical and Experimental Modelling of Microwave applicators (University of Cambridge, 1995).

[7] T. Sameshima, H. Hayasaka and T. Haba, Analysis of Microwave Absorption Caused by Free Carriers in Silicon, Japanese Journal of Applied Physics, 48, 2009, 021204.

[8] M. A. Green, Intrinsic concentration, effective densities of states and effective mass in silicon, Journal of Applied Physics, 67(6), 1990, pp 2944-2954.

[9] Y. P. Varshini, Temperature dependence of Energy gap in semiconductor, Physica, 34, 1967, pp 149.

[10] S. Reggiani, M. Valdinoci, L. Colalongo, M. Rudan, G. Baccarani, A. D. Stricker, F. Illien, N. Felber, W. Fichtner, and L. Zullino, Electron and Hole Mobility in Silicon at Large Operating Temperatures-Part I: Bulk Mobility, 49(3), 2002, pp 490499. 